\title{
All-optical Signal Processing of Fiber Impaiments in Dual-Polarization 112 Gbit/s m-ary QAM Coherent Transmission
}

\author{
Rameez Asif ${ }^{1 *}$, Muhammad Khawar Islam ${ }^{1}$, and Muhammad Zafrullah ${ }^{2}$ \\ ${ }^{I}$ Department of Telecommunication Engineering, University of Engineering and Technology (UET), \\ Taxila 47050, Pakistan \\ ${ }^{2}$ Department of Electronics Engineering, University of Engineering and Technology (UET), Taxila 47050, Pakistan
}

(Received August 23, 2012 : revised November 19, 2012 : accepted December 21, 2012)

\begin{abstract}
We have numerically implemented a receiver side all-optical signal processing method, i.e. optical backward propagation (OBP), by dispersion compensating fiber (DCF) and non-linear compensator (NLC) devised by effective negative Kerr non-linear coefficient using two highly non-linear fibers (HNLFs). The method is implemented for the post-processing of fiber transmission impairments, i.e. chromatic dispersion (CD) and non-linearities (NL). The OBP module is evaluated for dual-polarization (DP) m-ary $(\mathrm{m}=4,16,32,64,256)$ quadrature amplitude modulation (QAM) in $112 \mathrm{Gbit} / \mathrm{s}$ coherent transmission over $1200 \mathrm{~km}$ standard single mode fiber (SMF). We have also investigated an intensity limited optical backward propagation module (IL-OBP) by using a self-phase modulation-based optical limiter with an appropriate pre-chirping to compensate for the intensity fluctuations in the transmission link. Our results show that in highly non-linear sensitive 256QAM transmission, we have observed a $66 \%$ increase in the transmission distance by implementing IL-OBP as compared to conventional OBP.
\end{abstract}

Keywords : Fiber optic communication, Coherent transmission, Modulation, Single mode fiber OCIS codes : (060.0060) Fiber optics and optical communications; (060.4510) Optical communications

\section{INTRODUCTION}

Digital compensation of linear and non-linear signal distortions with backward propagation algorithm (DBP) is a topic of high interest in recent months, to improve the non-linear tolerance of optical communication systems. With higher baud-rates and advanced modulation formats, channel capacity $[1,2]$ is limited by the signal degradation due to fiber non-linearity, as it increases with signal input power. The DBP algorithm allows joint compensation of chromatic dispersion (CD) and non-linearities (NL) [3-6] along with the coherent receiver. It can be implemented by inversely solving the non-linear Schrodinger equation (NLSE) using split-step Fourier methods (SSFM). It is demonstrated that DBP in a single channel transmission can be improved by using a modified split-step Fourier method (M-DBP) $[7,8]$. M-DBP is based on the optimization of non-linear calculation point (NLCP). In order to reduce the complexity of the algorithm, the correlated DBP (CBP) algorithm is introduced in $[9,10]$. The performance of DBP algorithm is evaluated for diverse baud-rates i.e., 14Gbaud, 28Gbaud and 56Gbaud with QPSK modulation formats [11-14]. Especially with the introduction of logarithmic step-size based DBP algorithm (L-DBP) [12-14], which shows efficient system performance as compared to M-DBP, the nonlinear threshold point (NLT) of higher baud-rates transmissions are significantly improved. Due to exponentially growing global bandwidth demand, polarization-division multiplexed 16 state quadrature amplitude modulation (PDM 16-QAM) with high spectral efficiency (SE) is becoming a promising candidate to achieve per channel bit-rates beyond $100 \mathrm{Gbit} / \mathrm{s}$ and has been the subject of extensive research [15-19]. The multi-channel transmission performance over $670 \mathrm{~km}$ SMF and $656 \mathrm{~km}$ LA-PSCF fiber is reported in $[16,17]$. Whereas; the evaluation of DBP algorithm in $224 \mathrm{Gbit} / \mathrm{s}$ PM-16QAM system over $250 \mathrm{~km}$ of SMF with un-compensated link is reported in [19], with $3.5 \mathrm{~dB}$ of Q-improvement in the performance.

\footnotetext{
*Corresponding author: rameez@lhft.eei.uni-erlangen.de

Color versions of one or more of the figures in this paper are available online.
} 
However, this high SE is obtained at the expense of complicated transmitter and receiver structures, higher OSNR requirements and dense constellation diagrams, which collectively make them less robust against fiber transmission impairments i.e., chromatic dispersion (CD) and non-linearities (NL). But also the complexity of the DBP algorithm is extremely high due to ultra-wide band-width requirements that it becomes an intrinsic impediment in the real-time implementation of the DBP algorithm. To overcome the computational complexities of digital backward propagation, an alternative approach of optical backward propagation (OBP) is proposed [20]. The OBP module consists of dispersion compensating fiber (DCF) and non-linearity compensator (NLC). In the NLC part of the OBP, an effective negative Kerr non-linear coefficient using two highly non-linear fibers is realized. As a result NLC imparts a phase shift that is equal in magnitude to the non-linear phase shift due to fiber propagation, but opposite in sign, the same as the non-linear operator $(\mathrm{N})$ in digital backward propagation.

It is a well-known fact that in the all-optical signal processing techniques intensity fluctuation is a serious problem [21]. To date, numerous techniques for optical intensity stabilization including optical limiters, optical reshaping techniques and optical regenerators have been evaluated [21-23]. Among them, the techniques that are based on self-phase modulation (SPM) methods are more stable, having less complexity and supporting higher bit rates, i.e. $100 \mathrm{Gbit} / \mathrm{s}$ and beyond $[23,24]$. The SPM achieve limiting function by extracting the center frequency part of optical pulses after the SPM. After the input peak intensity reaches a certain threshold, the energy around the center frequency is spread out to both its frequency sides. As a result, the intensity around the center frequency can ideally keep balance between input and spread out energies. However, the behavior of the SPM is not ideal and the amount of spread out energy becomes larger or smaller than the input energy after a certain threshold point and intensity of optical pulses extracted starts varying [25]. To avoid this artifact, a stable SPM-based all-optical intensity limiter is proposed [26] based on the pre-chirping procedure. The intensity of center wavelength components depends on how the input pulse is chirped. Moreover, by adding an appropriate amount of chirp, the center wavelength intensity stabilization through a single SPM limiter can be achieved.

In this paper, we have numerically implemented optical backward propagation (OBP) and evaluated it for dual-polarization (DP) m-ary $(\mathrm{m}=4,16,32,64,256)$ quadrature amplitude modulation (QAM) in $112 \mathrm{Gbit} / \mathrm{s}$ coherent transmission over standard single mode fiber (SMF). OBP module jointly compensates $\mathrm{CD}$ and $\mathrm{NL}$ and gives enhanced system performance as compared to the electronic dispersion compensation (EDC), or in other words, linear equalization (LE) realized by the finite impulse response (FIR) filters. We have also investigated intensity limited optical backward propagation (IL-OBP) by using a self-phase modulation (SPM) based optical limiter with an appropriate pre-chirping to compensate for the intensity fluctuations in the transmission link. The IL-OBP gives improved system performance as compared to conventional OBP.

\section{NUMERICAL MODEL}

Figure 1 illustrates the numerical model of a single channel dual-polarization quadrature amplitude modulation (DP-QAM) based transmission system operated at a bit rate of $112 \mathrm{Gbit} / \mathrm{s}$ and at a wavelength of $1550 \mathrm{~nm}$. The data stream consists of $2^{16}-1$ pseudo-random binary sequence (PRBS). To simplify our numerical analysis and to focus on the deterministic transmission impairments, i.e. $\mathrm{CD}$ and $\mathrm{NL}$, we neglect the effect of polarization mode dispersion (PMD) and laser line width. We configured 5 transmission systems, i.e. DP-4QAM, DP-16QAM, DP-32QAM, DP-64QAM and DP-256QAM, operating at the baud rates of 28Gbaud, 14Gbaud, 11.2Gbaud, 9.33Gbaud and 7 Gbaud respectively. The signals are transmitted over $1200 \mathrm{~km}$ standard SMF

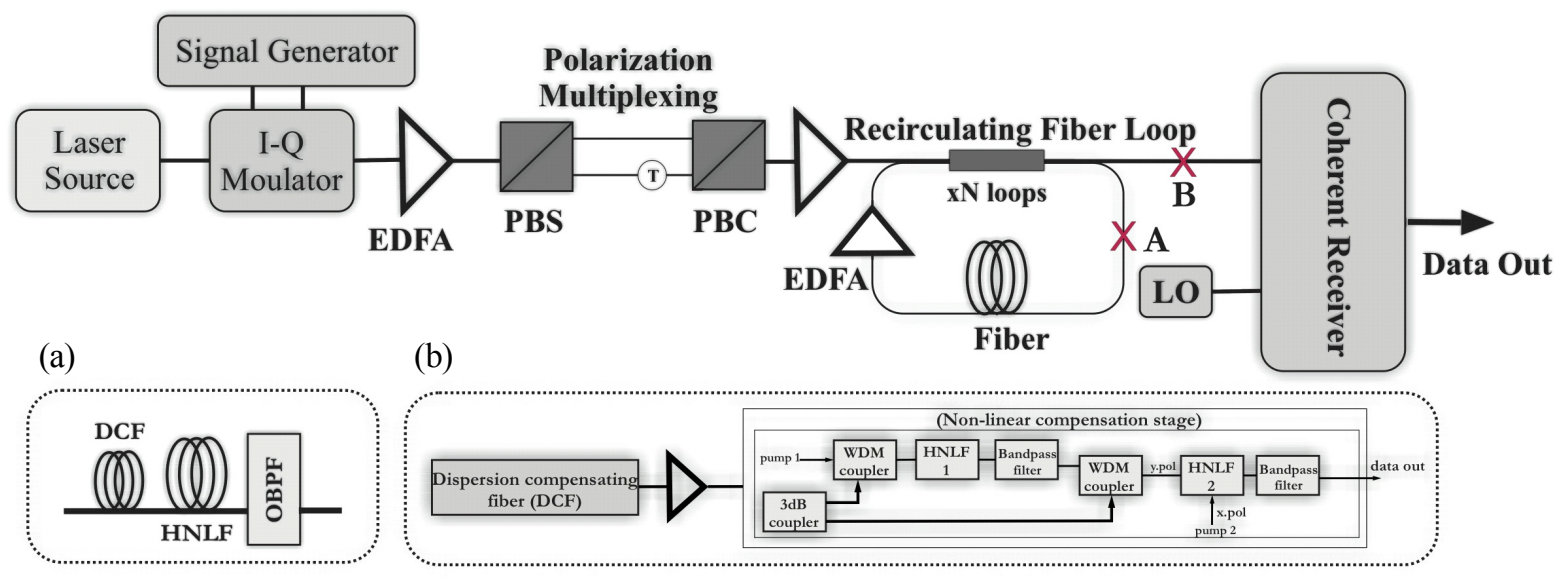

FIG. 1. Numerical model of DP m-ary QAM 112 Gbit/s coherent transmission (top). (a) SPM based all-optical intensity limiter and (b) Block diagram of OBP module. 
$(15$ spans $\times 80 \mathrm{~km})$ with physical parameters of dispersion $\mathrm{D}=16 \mathrm{ps} / \mathrm{nm}-\mathrm{km}$, non-linear coefficient $\gamma=1.3\left(\mathrm{~km}^{-1} \cdot \mathrm{W}^{-1}\right)$ and attenuation constant $\alpha=0.2 \mathrm{~dB} / \mathrm{km}$. No in-line optical dispersion compensating fiber (DCF) is used to compensate the chromatic dispersion. Erbium-doped fiber amplifiers (EDFAs) are modeled with $16 \mathrm{~dB}$ of gain and $4 \mathrm{~dB}$ of noise figure.

After processing the signals by the optical backward propagation module, a homodyne coherent, polarization and phase diversity receiver is used consisting of $2 \times 490^{\circ}$ optical hybrids followed by balanced detectors. The signal is sampled at two samples per symbol and passed on to the DSP module. An anti-aliasing $2^{\text {nd }}$ - order low pass (LP) Gaussian filter is also used at the receiver. The sampled signal goes through the DSP module having the adaptive polarization de-multiplexing which is realized by finite impulse response butterfly equalizer using the constant-modulus algorithm. It is assisted by the phase error estimation module based on the Viterbi-and- Viterbi algorithm which is able to remove small residual constellation rotations. When the chromatic dispersion is digitally compensated [electronic dispersion compensation (EDC)], finite impulse response (FIR) filters are used (T/2-spaced taps) and is adapted using a least mean square (LMS) algorithm. The transmission performances are compared by Q-factor $(\mathrm{dB})$ and bit-error-ratio (BER) analysis, whereas the Q-factor (dB) is directly calculated from BER by using BER = $\frac{1}{2} \operatorname{erfc} \frac{Q}{\sqrt{2}}$ and the FEC limit corresponds to $\mathrm{BER}=3.8 \times 10^{-3}$.

Figure 1(a) depicts the basic block diagram of the SPM-based all-optical intensity limiter which is placed in the main transmission system at point (A) for IL-OBP and removed for conventional implementation of OBP. This module consists of an EDFA, DCF, HNLF and an optical band-pass filter (OBPF), cascaded together [26]. An amplified optical signal is passed through a DCF to add the up-chirp in the signal. Then the signal enters in HNLF having the physical parameters of length $(\mathrm{L})=1.5 \mathrm{~km}, \mathrm{D}=0.5 \mathrm{ps} / \mathrm{nm}-\mathrm{km}$, $\gamma=11.5\left(\mathrm{~km}^{-1} . \mathrm{W}^{1}\right), \frac{d D}{d \lambda}=0.026 \mathrm{ps} / \mathrm{nm}^{2}-\mathrm{km}$ and $\alpha=0.7 \mathrm{~dB} / \mathrm{km}$.

The signals experience SPM providing spectral broadening, which maintains a constant intensity at the center wavelength because of the pre-chirping procedure. Finally the signals are filtered with the help of OBPF, which is a 2nd order Gaussian filter. The OBP module, as illustrated in Fig. 1(b) is numerically implemented by using the parameters given in [20]. The length of the DCF is adjusted according to the accumulative chromatic dispersion in the transmission link. The physical parameters of DCF are: $\mathrm{D}=-80 \mathrm{ps} / \mathrm{nm}-\mathrm{km}$, $\gamma=4.5\left(\mathrm{~km}^{-1} . \mathrm{W}^{-1}\right)$ and $\mathrm{a}=0.5 \mathrm{~dB} / \mathrm{km}$. The combination of HNLFs which are used to realize an effective negative Kerr non-linear coefficient are modeled with $\gamma=2000\left(\mathrm{~km}^{-1} . \mathrm{W}^{1}\right)$ and $\mathrm{L}=232 \mathrm{~m}$ for HNLF1 and $\gamma=1000\left(\mathrm{~km}^{-1} \cdot \mathrm{W}^{-1}\right)$ and $\mathrm{L}=200 \mathrm{~m}$ for HNLF2. The pump powers are $10 \mathrm{~mW}$ and $5.6 \mathrm{~mW}$ for pump1 and pump2 respectively. The effective lengths of the HNLFs are optimized according to the modulation format used in order to get the maximum transmission performance through efficient compensation of fiber nonlinear impairments. All the numerical simulations are carried out by using Optisystems v10 and MATLAB v2011b.

\section{RESULTS AND DISCUSSIONS}

Figure 2 depicts the comparative analysis of EDC, optical backward propagation (OBP) and intensity limited optical backward propagation (IL-OBP). The results of single channel $112 \mathrm{Gbit} / \mathrm{s}$ DP-64QAM transmission over $1200 \mathrm{~km}$ standard single mode fiber are as shown in Fig. 2. The system performance is quantified by means of bit-error-ratio (BER) as a function of single launch power. The results depict improved system performance in both the cases of optical backward propagation (OBP), as compared to EDC, which shows efficient joint compensation of $\mathrm{CD}$ and NL.

Firstly, the conventional OBP is numerically investigated for the above mentioned transmission system. The maximum system performance is achieved at a signal launch power of $-2 \mathrm{dBm}$. The non-linear threshold point (NLT) is improved by $4 \mathrm{dBm}$ as compared to EDC. Then as a next step, IL-OBP is numerically investigated which was realized by implementing an SPM based all-optical intensity limiter. This scheme has further improved the system performance by suppressing the intensity fluctuations in the transmission link. The system performance by using IL-OBP is improved by a factor of $1.9 \mathrm{~dB}$ of signal input power for same reference amount of BER for OBP at NLT point. The implementations of both the OBP techniques correspond to the implementation of compensating fiber transmission impairments in one step for the whole transmission link. Whereas, for DP-64QAM transmission system to get the efficient system performance by DBP, 40 step per fiber span were processed [18]. This means that OBP concept has a very low complexity and can be used as a real time

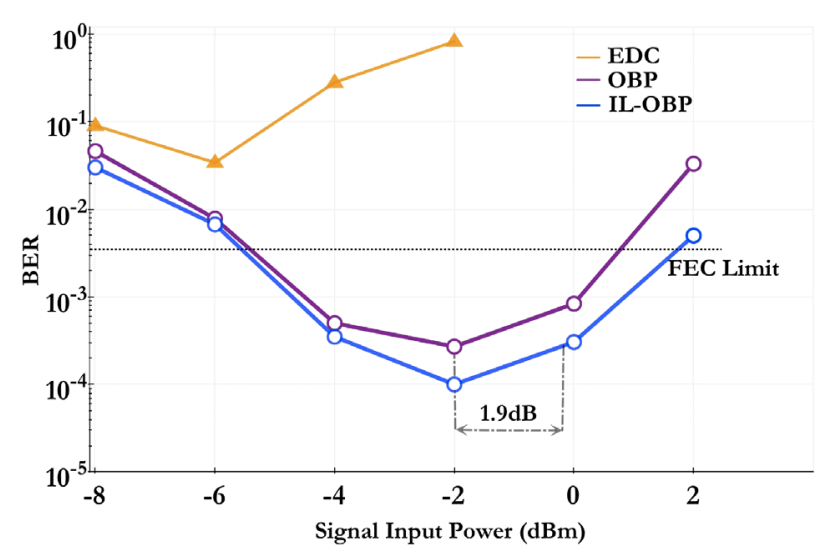

FIG. 2. BER as a function of signal input launch power for $112 \mathrm{Gbit} / \mathrm{s}$ DP-64QAM over $1200 \mathrm{~km}$ SMF by using EDC, OBP and IL-OBP. 
signal processing modules with coherent receivers. Fig. 3 qualitatively depicts, in-terms of constellation diagrams, the performance comparison of OBP and IL-OBP at the non-linear threshold point (NLT) and also the corresponding performance of EDC for the same transmission distance for 112 Gbit/s DP m-ary QAM ( $m=4,16,32,64,256)$. The results show improved system performance and enhanced transmission distance of IL-OBP as compared to conventional OBP. As mentioned earlier, in our numerical analysis we neglect the PMD effect, which is a stochastic process, and we are emphasizing on the compensation of deterministic fiber impairments, i.e. CD and NL. Otherwise due to PMD, polarization dependent loss can effect the performance of the system and it is necessary to have polarization tracking or in other words the polarization alignment between pumps and signal in all-optical signal processing methods.

To estimate the maximum system performance by implementing EDC, OBP and IL-OBP, we have evaluated the Q-factor $(\mathrm{dB})$ as a function for diverse transmission distances for all the modulation formats, as shown in Fig. 4. It can be seen from the results that DP- 4QAM transmission has the maximum reach in terms of transmission distance in $\mathrm{km}$. Furthermore, the results from OBP and IL-OBP also complement the fact that transmission reduces by a factor of $\approx 3$ times for a 2 unit increase in capacity $(\log 2 \mathrm{~m})$. The results in Fig. 4 show additional reduction of transmission distance by a factor of 0.5 as compared to the results reported in [18]. This is due to the fact of DCF induced discrepancies in $\mathrm{OBP}$, while all the $\mathrm{CD}$ is compensated digitally in DBP. Therefore the launch power to the DCF is kept low in this numerical analysis so that the non-linear effects in the DCF can be negligibly small.

We have further summarized and compared the improvement in the transmission performance for each modulation for $112 \mathrm{Gbit} / \mathrm{s}$ DP m-ary QAM $(\mathrm{m}=4,16,32,64,256)$ transmission achieving a minimum BER of $\approx 10^{-3}$. The results are as shown in Fig. 5, where the largest system improvements (when OBP and IL-OBP are compared with EDC) are depicted in higher-order modulation formats. This is

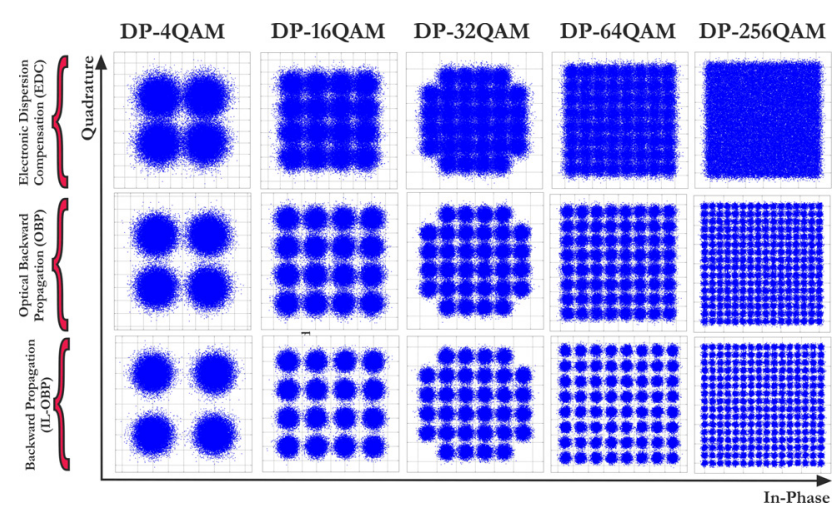

FIG. 3. Constellation plots after: EDC, OBP and IL-OBP for $112 \mathrm{Gbit} / \mathrm{s}$ DP m-ary QAM $(\mathrm{m}=4,16,32,64,256)$ at NLT point. due to the fact of reduced dispersive effects in higher order modulation formats with lower baud rates as discussed in the numerical model section. To summarize, the DP256QAM transmission attains a transmission distance of $580 \mathrm{~km}$ with OBP for a minimum BER of $\approx 10^{-3}$ as compared to $960 \mathrm{~km}$ with IL-OBP, which depict $\approx 66 \%$ increment in maximum reach. The overall results depict that IL-OBP performs efficiently with higher order modulation formats, i.e. DP-64QAM, DP-256QAM, by suppressing intensity fluctuations or in other words limiting amplitude noise in the transmission link.

\section{CONCLUSION}

In this paper, we have discussed the transmission performance of optical backward propagation (OBP) for 112 Gbit/s DP m-ary QAM $(\mathrm{m}=4,16,32,64,256)$ system over SMF fiber. The results depict efficient compensation of $\mathrm{CD}$ and NL and shows better performance as compared to

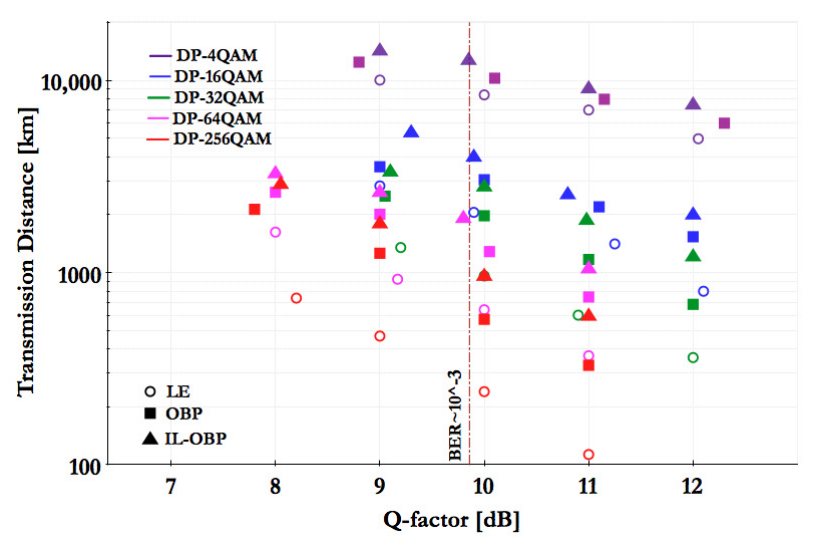

FIG. 4. Q-factor $[\mathrm{dB}]$ as a function of various transmission distances for $112 \mathrm{Gbit} / \mathrm{s}$ DP m-ary QAM (m=4,16,32,64,256) transmission.

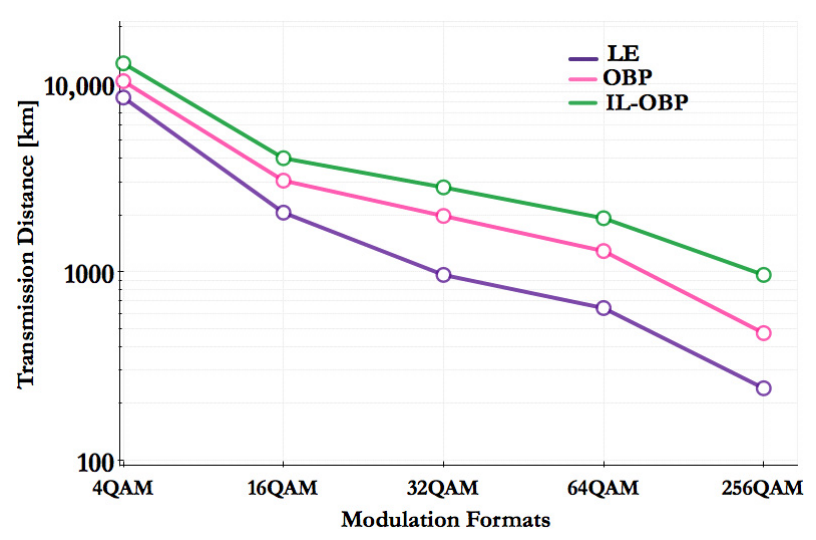

FIG. 5. Maximum transmission distance at the NLT point for each modulation for $112 \mathrm{Gbit} / \mathrm{s}$ DP m-ary QAM $(\mathrm{m}=4,16,32,64,256)$ transmission achieving a minimum BER of $\approx 10^{-3}$. 
EDC over the range of modulation formats listed above. We have further demonstrated that the additional improvement in system performance can be obtained with a self-phase modulation (SPM) based optical limiter with an appropriate pre-chirping to compensate for the intensity fluctuations in the transmission link. The results depict that in DP-256QAM transmission, we have observed a $66 \%$ increase in the transmission distance by implementing IL-OBP as compared to conventional OBP. From the results, it is evident that this scheme of fiber transmission impairment compensation with very low complexity can be used as a real time signal processing modules with coherent receivers as compared to DBP, where the computational effort and bandwidth required for higher order modulation formats would be prodigious.

\section{ACKNOWLEDGMENT}

The author gratefully acknowledge the technical support from Prof. Dr. Bernhard Schmauss and M.Sc. Chien-Yu Lin during the research stay at University of ErlangenNuremberg, Germany and also the funding of the Erlangen Graduate School in Advanced Optical Technologies (SAOT) by the German National Science Foundation [Deutsche ForschungsGemeinschaft, (DFG)] in the framework of the excellence initiative.

\section{REFERENCES}

1. C. R. S Fludger, T. Duthel, D. van den Borne, C. Schulien, E. Schmidt, T. Wuth, J. Geyer, E. de Man, G. D. Khoe, H. de Waardt, "Coherent equalization and POLMUXRZ-DQPSK for robust 100-GE transmission," IEEE J. Lightwave Technol. 26, 64-72 (2008).

2. P. P. Mitra and J. B. Stark, "Non-linear limits to the information capacity of optical fibre communications," Nature 411, 1027 1030 (2001).

3. E. Ip and J. M. Kahn, "Compensation of dispersion and non-linear impairments using digital backpropagation," IEEE J. Lightwave Technol. 26, 3416-3425 (2008).

4. D. S. Millar, S. Makovejs, C. Behrens, S. Hellerbrand, R. Killey, P. Bayvel, S. J Savory, "Mitigation of fiber nonlinearity using a digital coherent receiver," IEEE J. Select. Topics Quantum Electron. 16, 1217-1226 (2010).

5. F. Yaman and G. Li, "Non-linear impairment compensation for polarization-division multiplexed WDM transmission using digital backward propagation," IEEE Photonics Journal 2, 144-152 (2009).

6. R. Asif, C. Y. Lin, M. Holtmannspoetter, and B. Schmauss, "Optimized digital backward propagation for phase modulated signals in mixed-optical fiber transmission link," Opt. Express 18, 22796-22807 (2010).

7. C. Y. Lin, M. Holtmannspoetter, R. Asif, and B. Schmauss, "Compensation of transmission impairments by digital backward propagation for different link designs," in Proc. 36th European Conference Optical Communication (ECOC) (Torino Italy, September 2010), paper 3.16.

8. L. Du and A. Lowery, "Improved single channel back-propagation for intra-channel fiber non-linearity compensation in long-haul optical communication systems," Opt. Express 18, 17075-17088 (2010).

9. L. Li, Z. Tao, L. Dou, W. Yan, S. Oda, T. Tanimura, T. Hoshida, and J. Rasmussen, "Implementation efficient nonlinear equalizer based on correlated digital back-propagation," in Proc. Conference on Optical Fiber Communication/National Fiber Optic Engineers Conference (OFC/NFOEC) (Los Angeles, USA, March 2011), paper OWW3.

10. D. Rafique, M. Mussolin, M. Forzati, J. Martensson, M. N. Chugtai, and A. Ellis, "Compensation of intra-channel nonlinear fibre impairments using simplified digital back-propagation algorithm," Opt. Express 19, 9453-9460 (2011).

11. P. Poggiolini, G. Bosco, A. Carena, V. Curri, V. Miot, and F. Forghieri, "Performance dependence on channel baud-rate of PM-QPSK systems over uncompensated links," IEEE Photon. Technol. Lett. 23, 15-17 (2011).

12. R. Asif, C. Y. Lin, M. Holtmannspoetter, and B. Schmauss, "Logarithmic step-size based digital backward propagation in N-channel $112 \mathrm{Gbit} / \mathrm{s} / \mathrm{ch}$ DP-QPSK transmission," in Proc. 13th International Conference on Transparent Optical Networks (ICTON) (Stockholm, Sweden, June 2011), paper T.u.P6.

13. R. Asif, C. Y. Lin, and B. Schmauss, "Impact of channel baud-rate on logarithmic digital backward propagation in DP-QPSK system with uncompensated transmission links," Opt. Commun. 284, 5673-5677 (2011).

14. R. Asif, C. Y. Lin, M. Holtmannspoetter, and B. Schmauss, "Multi-span digital non-linear compensation for dual-polarization quadrature phase shift keying Long-Haul communication systems," Opt. Commun. 285, 1814-1818 (2012).

15. M. Nolle, J. Hilt, L. Molle, M. Seimetz, and R. Freund, "8×224 Gbit/s PDM 16QAM WDM transmission with real-time signal processing at the transmitter," in Proc. 36th European Conference and Exhibition on Optical Communication (ECOC) 2010 (Torino, Italy, September 2010), paper We.8.C.4.

16. M. S. Alfiad, M. Kuschnerov, S. L. Jansen, T. Wuth, D. van den Borne, and H. de Waardt, " $11 \times 224-\mathrm{Gb} / \mathrm{s}$ POLMUXRZ- 16QAM transmission over $670 \mathrm{~km}$ of SSMF with 50-GHz channel spacing," IEEE Photon. Technol. Lett. 22, 1150-1152 (2010).

17. V. Sleiffer, M. S. Alfiad, D. van den Borne, M. Kuschnerov, V. Veljanovski, M. Hirano, Y. Yamamoto, T. Sasaki, S. L. Jansen, T. Wuth, and H. de Waardt, "10×224-Gb/s POLMUX-16QAM transmission over $656 \mathrm{~km}$ of large Aeff PSCF with a spectral efficiency of $5.6 \mathrm{~b} / \mathrm{s} / \mathrm{Hz}$," IEEE Photon. Technol. Lett. 23, 1427-1429 (2011).

18. D. Rafique, J. Zhao, and A. Ellis, "Digital back-propagation for spectrally efficient WDM $112 \mathrm{Gbit} / \mathrm{s}$ PM m-ary QAM transmission," Opt. Express 19, 5219-5224 (2011).

19. M. Mussolin, D. Rafique, J. Martensson, M. Forzati, J. Fischer, L. Molle, M. Nlle, C. Schubert, and A. Ellis, "Polarization multiplexed $224 \mathrm{~Gb} / \mathrm{s}$ 16QAM transmission employing digital back-propagation," in Proc. 37th European Conference and Exposition on Optical Communications, OSA Technical Digest (CD) (Geneva, Switzerland, 2011), 
paper We.8.B.6.

20. S. Kumar and D. Yang, "Optical backpropagation for fiber-optic communications using highly nonlinear fibers," Opt. Lett. 36, 1038-1040 (2011).

21. M. Matsumoto, "Fiber-based all-optical signal regeneration," IEEE J. Select. Topics Quantum Electron. 18, 738-752 (2012).

22. M. Matsumoto and K. Sanuki, "Performance improvement of DPSK signal transmission by a phase-preserving amplitude limiter," Opt. Express 15, 8094-8103 (2007).

23. M. Gay, M. Costa e Silva, T. N. Nguyen, L. Bramerie, T. Chartier, M. Joindot, J. C. Simon, J. Fatome, C. Finot, and J. L. Oudar, "Bit-error-rate assessment of $170-\mathrm{Gb} / \mathrm{s}$ regeneration using a saturable absorber and a nonlinear-fiber- based power limiter," IEEE Photon. Technol. Lett. 22, 158-160 (2010).
24. T. Ohara, H. Takara, S. Kawanishi, T. Yamada, and M. M. Fejer, "160 GBit/s all-optical limiter based on spectrally filtered optical solitons,” IEEE Photon. Technol. Lett. 16, 2311-2313 (2004).

25. T. Konishi, H. Goto, T. Kato, and K. Kawanishi, "Advanced optical limiting function based on effective understanding of physical phenomena," in Proc. 11th International Conference on Transparent Optical Networks (ICTON) (Stockholm, Sweden, July 2009), pp.1-4.

26. K. Kawanishi, F. Drouet, K. Itoh, and T. Konishi, "Highly accurate compensation technique for $10-\mathrm{GHz}$ pulse intensity fluctuation using spm-based all-optical intensity limiter," IEEE Photon. Technol. Lett. 24, 119-121 (2012). 